\title{
The 6204 Bearing Dynamic Analysis Based on UG NX Technology
}

\author{
Zhao Song \\ Department of Mechanical and Electrical Engineering Xijing University, Xian 710123,China \\ 634034944@qq.com
}

Keywords: Bearing; UG NX; Dynamic Analysis.

Abstract. This paper based on the 6204 rolling bearing as the research object, Application of UG NX software to carry on the analysis to the bearing. In the modeling module of the UG NX, drawing the bearings, shaft, gears and fit them together. When the move correctly, take the proper load on it and make the software calculate the transmission shaft of the mechanism movement through the shaft to the bearing. In the advanced simulation module of UG NX simplify the bearing. Then the finite element mesh for the simplified bearing. Add the load calculated in the dynamic simulation on the intersected bearing. Solve it by the nonlinear calculation scheme of UG NX. Get the stress cloud map and the maximum stress value and the maximum stress value position of the each fitting with the specific load. Providing the theory evidence for the structural design of bearing.

\section{Introduction}

Since the late 1970s, the international large-scale construction of the finite element program has reached several hundred kinds. Its advanced simulation module provides many kinds of computing devices, including NASTRAN MSC, NASTRAN ANSYS, NX, UNV IDEAS and ABAQUS,Among them, the NX NASTRAN solver is most widely used, through years of development and constantly upgrade version, the analysis is more and more species, the solution function is more and more strong, the result has become the aerospace vehicle industry, CAE standard, FAA certification..

\section{Three dimensional model of bearing}

According to the finite element analysis of the actual needs, to ignore the 6204 parameters of [1] bearing clearance. When ignoring the main structural parameters of 6204 parameters after the clearance of bearing table1

Table1 6204 the main structural parameters of the bearing

\begin{tabular}{c|c|c}
\hline Spare parts & Structure parameter & Size $(\mathrm{mm})$ \\
\hline \multirow{2}{*}{$\begin{array}{c}\text { The inner } \\
\text { ring }\end{array}$} & Groove curvature radius ri & 4.088 \\
\cline { 2 - 3 } & Internal channel diameter di & 26.062 \\
\cline { 2 - 3 } & Edge height diameter d2 & 28.8 \\
\hline \multirow{2}{*}{$\begin{array}{c}\text { The outer } \\
\text { ring }\end{array}$} & Groove curvature radius Re & 4.167 \\
\cline { 2 - 3 } & Outer channel diameter De & 41.938 \\
\cline { 2 - 3 } & Edge height diameter D2 & 39.2 \\
\hline
\end{tabular}

According to the structure parameters of the 6204 bearing given in Table 1, the CAD module of the UG NX software is used to establish the three-dimensional model of the 6204 ball bearing. The results are shown in Fig. 1.

Because the 6204 supporting structure is used to improve the efficiency of the finite element analysis, the computer resource is saved, and the symmetry of the 1/4 model is taken as the finite element analysis, as shown in Fig. 2, Then, the finite element analysis model of 6204 ball bearings can be established. 


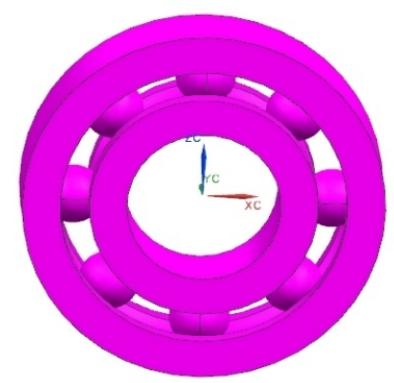

Fig. 1 three-dimensional model

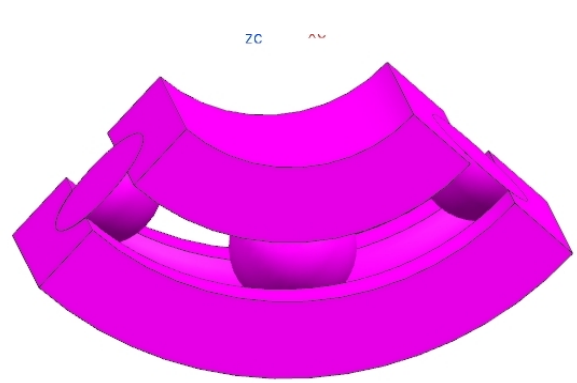

fig.2 analysis model

\section{Simulation analysis}

(1)Division of finite element mesh of 6204 bearing.

Analysis is to use the nonlinear analysis method, so the solution is relatively complex, and analysis in order to make the cell simplification, so it is necessary to split the remaining $1 / 4$ of the bearings, as shown in Figure 3-3. This segmentation can make bearing finite element in the non contact place in segmentation when a little, the solution is simple, finite element is smaller but also can make the bearing segmentation at the contact place, making the results accurate. After dividing the geometry, the first assignment of material, click the toolbar in the material properties, in which the selection of materials for G15. In order to define the physical properties of the bearings, you need to select the "physical properties" button in the toolbar, call the physical property table Manager dialog box. After that, you need to create a physical unit, the unit's property is selected as "PSOLID", the meaning of which is "body unit". And then to create the "body unit" of the property, material selection to choose the material to be defined, the other is the default value of the system. Select the required binding surface to match, and the final results are shown in Fig.3. This command represents the same on the geometric structure of the pair, and can automatically merge the two faces to create a single face that is shared by a single pair.

After the grid to collect, select the toolbar of the grid trap pop-up dialog box, The default "unit topology" column of the various options in the content, in the "Property Solid" list box to select the set of "PSOLID1", the default grid name "Solid (1)". 9 individuals were selected to define the size of the grid in the cell size, in which the 4 individuals were exposed to make the definition of the cell smaller, so that the results can be accurate.

The results obtained are shown in Fig.4. Then click the test model button, check whether the cell division is wrong, check the error after you can enter the simulation analysis.

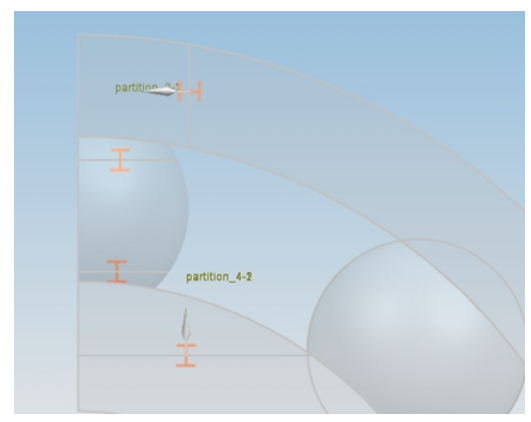

Fig.3 Bonded to the opposite

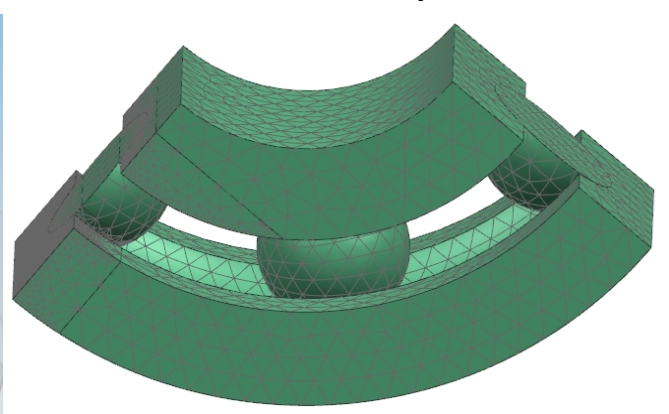

Fig.4 Grid partition result

(2) Finite element simulation analysis of 6204 bearing

Firstly, a new simulation, simulation in dialog box pop-up solution selected NX NASTRAN algorithm the most commonly used device, because the bearing ball and the inner and the outer contact is nonlinear, so the computation module can select the "ADVNL601106" type of nonlinear program. Then you need to create a constraint type, click the "constraint type" icon in the toolbar, select a fixed constraint, and then select the outer ring of the bearing to make it secure. Since the analysis of the bearings is only $1 / 4$, so the two sides need to make the approximation with the symmetry constraints 
into an overall bearing, as shown in Fig.5. After the constraint is added to define the load, in the toolbar of the "load type" in a direct bearing on the choice, in the above input predefined bearing force, as shown in Fig.6. Finally, the key point of contact is defined, and the contact problem is a complex process. The determination of the boundary point of the contact state is the basic problem of the contact problem solving. The judgement conditions of the contact interface include normal and tangential conditions.

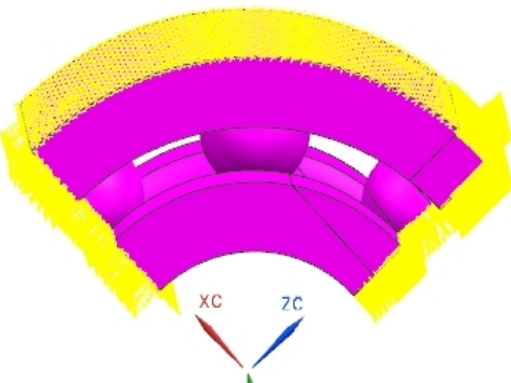

Fig.5 Bearing constraint

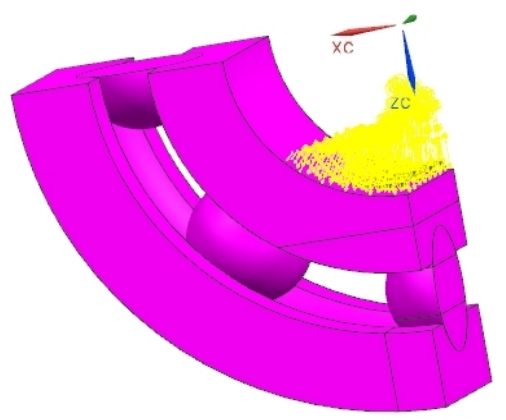

Fig.6 Bearing load

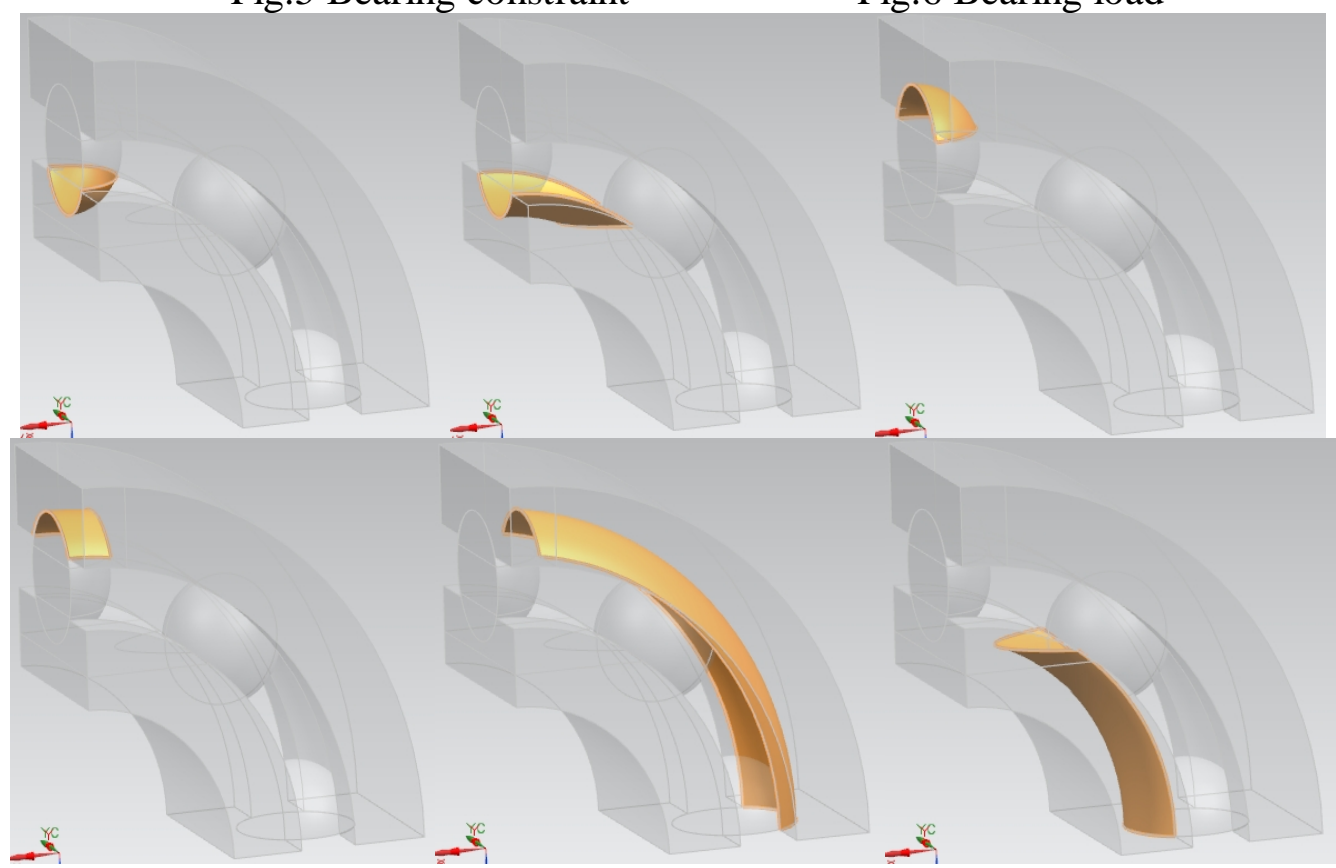

Fig.7 Divided region

As the bearing ball and inner ring is split, therefore, in the definition of contact area of the well selected, ahead of the best first definition of several areas of good contact: two no ball is split into two regions are defined, a segmented ball in two sides and above the bearing contact respectively. Defined as the two regions, and then because of the bearing inner and outer rings are divided into two parts, it is defined as four regions, dividing eight as shown in Fig.7 good areas. Then enter the toolbar of the "simulation object type" option, select the "face to face", in the pop-up dialog box select the source area and the target area, respectively, to select the region has been defined. Note that the contact area should be in accordance with the actual contact.

\section{Solution and result of 6204 bearing solution based on NX UG}

After solving, we can see the finite analysis of the process of nonlinear load history and the load of the number of steps, as shown in Fig.8. 


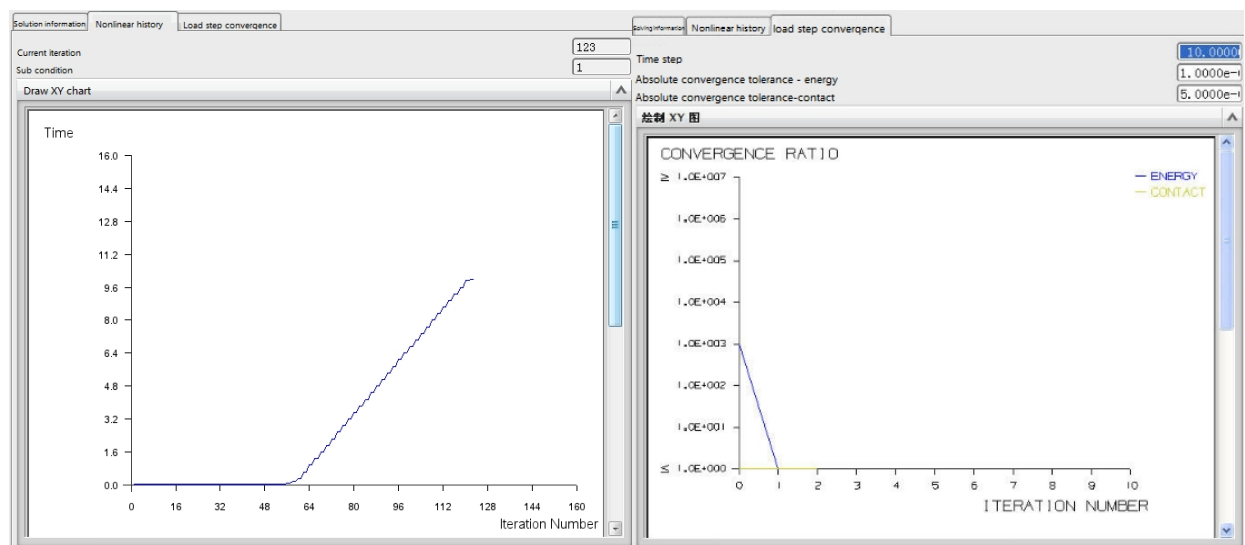

Fig.8 Nonlinear historical records and the number of load convergence steps

Enter the "post processing navigator" module, click the options in the specific values of force and deformation which can check each node or each unit, also can analyze and check to the adjacent or along one direction on each node or element deformation and stress change trend. Firstly, the finite element analysis results of 6204 bearing are analyzed, as shown in Fig.9. This graph can show that in dynamic analysis under the load, the maximum contact stress of $987.03 \mathrm{MPa} 6204$ rolling bearings, ball bearing contact stress distribution is small, the stress mainly distributed in the largest load to bear ball contact with the outer ring position, the contact area is elliptical shape; and the location of the contact stress, the stress distribution is very uneven, which is exactly in line with the rolling ball bearing contact stress distribution.

Then can analyze 6204 rolling bearing parts of the contact stress distribution diagram, in the analysis of the corresponding parts of the other components to hide out, as shown in Fig.10. The map shows that the bearing parts stress distribution diagram, can be seen that the maximum stress of the bearing's inner circle is $987.03 \mathrm{MPa}$, the maximum stress of the outer ring is $530.44 \mathrm{MPa}$, the maximum stress of the steel ball is $626.26 \mathrm{MPa}$. This shows that in the bearing force only under the condition of this case and the size of model establishment, the inner and outer contact stress is greater than the ball, it also shows the inner stress is much worse than the outer ring.

The finite element analysis results in failure analysis of bearing performance and with a lot of bearing failure, the main reason is the fatigue spalling of the inner ring, and a small ball minor failure, the outer ring from the surface can not see any damage, which proved that the finite element analysis method and results.

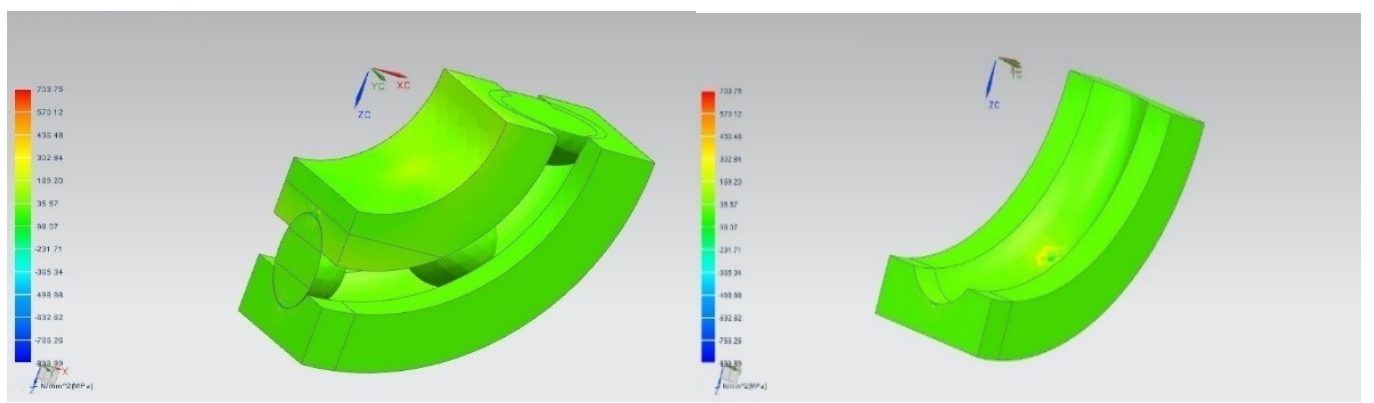

Fig.9 Contact stress distribution diagram

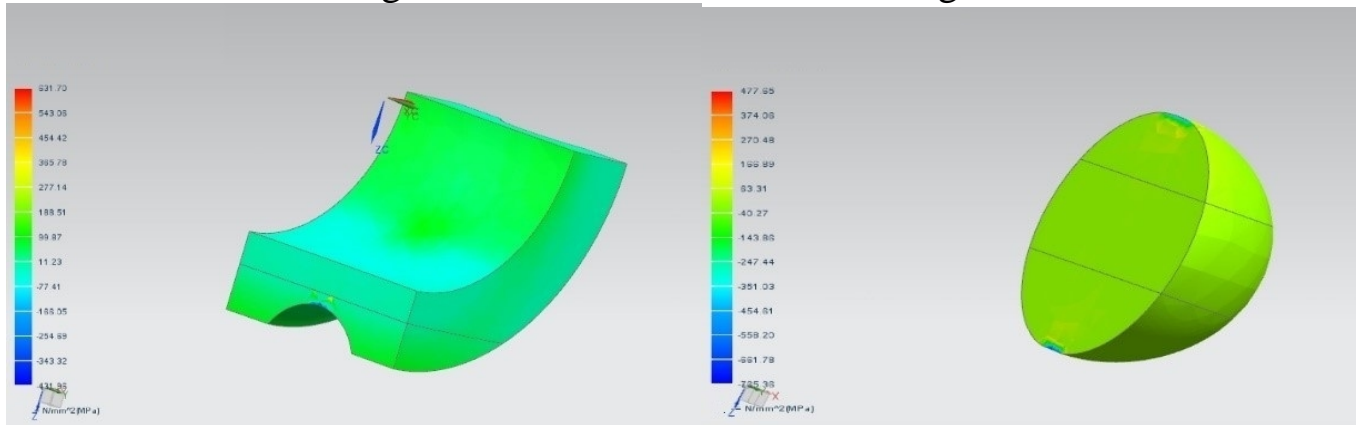

Fig.10 Stress distribution of inner and outer rings and balls 
Fig.11 the inner and outer stress distribution curves, seen from the stress distribution curve of contact stress, no obvious peak value, the maximum value distribution in a relatively wide area, and outside this area, the stress decreases rapidly, almost without force, showing the inner and outer rings raceway contact stress has obvious non-linear characteristic, also shows that the inner and outer rings under high contact stress, localized elastic deformation, changed from point contact to the contact area (small)

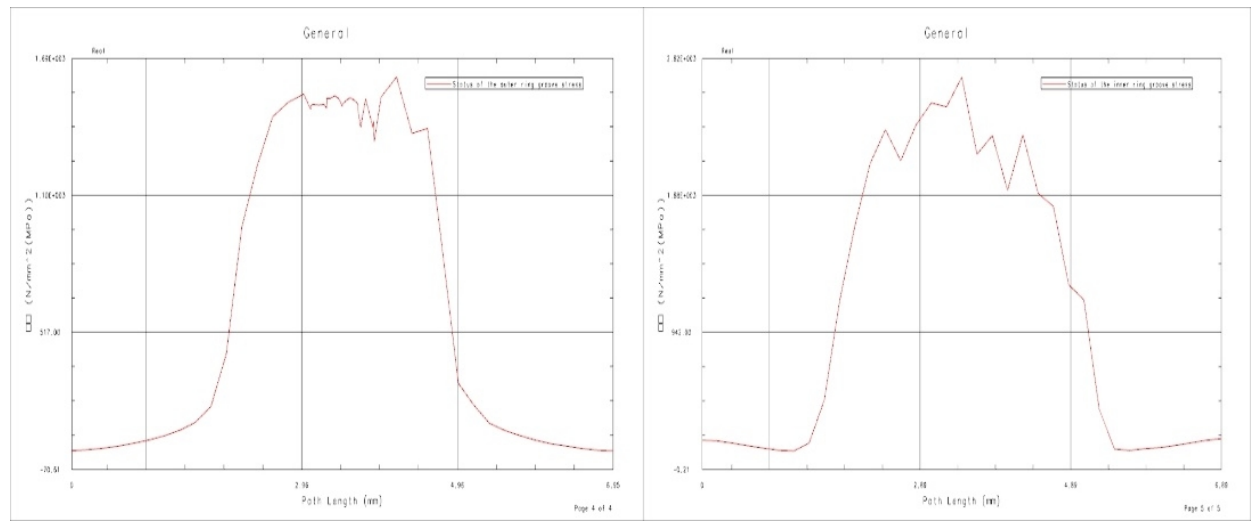

Fig.11 Stress distribution curve of inner and outer rings

According to the above analysis method of finite element, and then consider the actual bearing working conditions, such as the impact of the 6204 rolling bearings caused by centrifugal force in the dynamic condition (caused by stress increased, inner stress of contact, adjust the inner ring outer ring), or change the curvature coefficient of outer ring raceway curvature coefficient, or circle of curvature the adjustment coefficient and the realization of the inner and outer rings of equal contact stress design (such as life design goals)

\section{References}

[1] J. Guixi, Design analysis and experimental study of precision ball bearing based on magnetron sputtering ion plating technique, Xi'an University of technology(2011)

[2] S. Xue: The needle roller bearings analysis of a tracked ored vehicle suspension ,Shenyang Ligong University(2009).

[3] S. Xiaotian. Analysis of Forming Defects and The Mold Design of Shaft Part with Cross Wedge Rolling Process, College of Material Science and Engineering of Chongqing(2012).

[4] C. Xidong,Y. jie, Z. Xiaodong and F. Xiqiu: Development status and application of finite element method, Information of manufacturing industry in China, 2010(11): 28-40. 